\title{
Erratum to: Study of poly (sodium-4-styrenesulfonate) behavior in water/non-solvent mixtures by conductivity and refractive index measurements
}

\author{
Ons Zoghlami ${ }^{1,2} \cdot$ Moez Guettari ${ }^{1} \cdot$ Tahar Tajouri $^{1}$
}

Published online: 10 July 2017

(C) Springer-Verlag GmbH Germany 2017

Erratum to: Colloid and Polymer Science

DOI 10.1007/s00396-017-4104-y

The original version of this article unfortunately contained a mistake. The version of the article that was published online is not updated. The original article was corrected.

The online version of the original article can be found at http://dx.doi.org/ 10.1007/s00396-017-4104-y

Tahar Tajouri

tajourit2001@yahoo.fr

1 NMR in Polymers and Composites Laboratory, Université de Tunis IPEIT - UR 0113/04, 2, rue Jawhar Nahrou - Montfleury,

1008 Tunis, Tunisia

2 Faculty of Sciences, University of Tunis El Manar, El Manar, 2092 Tunis, Tunisia 